\title{
Connecting Bridges across the Digital Divide
}

\author{
William D. Tucker \\ Dept. of Computer Science, University of Cape Town \\ Private Bag, Rondebosch 7701 South Africa \\ btucker@cs.uct.ac.za \\ +27824948884
}

\begin{abstract}
Connecting people across the Digital Divide is as much a social effort as a technological one. We are developing a community-centered approach to learn how interaction techniques can compensate for poor communication across the Digital Divide. Preliminary trials have yielded interfaces that deal with poor quality by adapting Instant Messaging techniques for multiple modalities, providing improved semi-synchronous communication. Lessons learned suggest new ways to design user interfaces specifically for the developing world.
\end{abstract}

\section{Author Keywords}

Community-centered, Digital Divide, Instant Messaging, multi-modal, semi-synchronous, user interface.

\section{ACM Classification Keywords}

H.5.2.q. User-centered design.

\section{INTRODUCTION}

The "Digital Divide" is the growing gap that exists between those who have access to the resources of the global information revolution and those who are deprived of such access due to gaps in their education, personal handicap, poor digital infrastructure, or lack of advanced computer equipment. Bridging the Digital Divide is the effort to provide increased access to information and communication to those who have little or none at all. "Communication bridges" involve social dynamics as well as the technological tools that support social interaction. Our community-centered approach has produced innovative systems that provide completely new solutions to the issues that arise in building communication bridges. We support our user communities with new communication systems that are adapted to their requirements. We are developing a methodology to support this design process as well. We have found that sophisticated bridging systems impose delays upon the communication process. Additionally, the unreliable nature of the infrastructure also can result in extended breaks in communication. Thus, compensating for delay is of major importance in building automatic communication bridges over the Digital Divide.

\section{DIGITAL DIVIDE OPPORTUNITIES FOR HCI}

South Africa provides an interesting test bed for Digital Divide research. First world and developing communities exist side-by-side. Text, voice and video communications over the Internet Protocol (IP) offer many potential bridges over this divide. Yet these technological bridges are hampered by literacy and infrastructure disparities based ultimately on poverty. Even where Information and Communication Technology (ICT) is made available to the "masses", e.g. a telecentre in a rural area, other factors, such as poor network connectivity and erratic power supply usually render ICT unusable. Additionally, connectivity is simply too expensive to be financially sustainable in poverty-stricken communities. Many of these problems appear intractable. However, some can be overcome with Human Computer Interface (HCI) techniques. While there is ample research into the role of ICT in the developing world, e.g. IFIP WG 9.4, little of this research relates to HCI. Therefore, answers to the following research questions offer contributions to the field of HCI:

- How can HCI techniques bridge the Digital Divide?

- How can HCI techniques compensate for delay in Digital Divide situations?

- How do HCI techniques differ for both sides of a bridge over the Digital Divide?

- How can we measure the efficacy of these techniques?

SEMI-SYNCHRONOUS MULTI-MODAL MESSAGING

Interface techniques of text-based messaging offer answers to some of these questions. Instant messaging (IM) handles low-bandwidth situations, can "feel" real-time, and provides rather rich social connectivity [4, 6]. IM compensates for variable delay with interaction mechanisms such as presence and awareness, providing a connection between parties, even when not exchanging messages. Message persistence also contributes to a connection between parties. These connections appear to overcome the ebb and flow of asynchronous exchanges. However, text may not be the only desired form of interaction. It would be useful, therefore, to extend the IM interface mechanisms to include other modalities, such as voice and video. To do this, the underlying infrastructure must transparently handle both synchronous and asynchronous exchange at the transport protocol level for each modality. The user interface presents the challenge of applying IM interface techniques to non-text modalities, e.g. visualizing voice $[1,8]$.

\section{METHODOLOGY}

We believe a user-centered approach is most appropriate to address these issues. Application and interface requirements can be fed into an iterative loop that drives the design and development of the interaction, and indirectly, the underlying transport infrastructure. However, in the devel- 
oping world, the technological requirements exist within a complex web of other needs. End-user participation in the process can be problematic. Misinterpretation (on both sides) and unexpected needs are common. A wide, and culturally sensitive, view on the software process is required [3]. Therefore, we included other stakeholders, such as non-governmental organizations (NGOs) and other researchers already involved with a particular community. Rather than focus on particular individuals, we engage an entire community and therefore, we must always be aware of social subtleties. We call this a community-centered approach.

A measurement system operates alongside the technology development cycle. Starting with an initial baseline, participants are surveyed to determine how they tolerate problems with their communication systems. Further measurements are made as subsequent software modifications are introduced in the field. The software is also instrumented to record actual activity metrics, such as latency. These metrics are correlated with participants' subjective experiences to give a quantitative measure of how well the interaction mechanisms compensate for problems with the bridge.

\section{ADVANCE STATUS}

Field trials are underway with two communities in South Africa. The Bastion Centre for the Deaf serves a "disadvantaged" community in Cape Town that is marginalized from mainstream communications due to both poverty and hearing disorders. Voice/text relay enables a deaf person to use a "text telephone" to communicate with someone on a normal telephone via an operator with both devices. Because this service is not available in South Africa, we have built an automated voice/text relay system based on web services. Automated Speech Recognition (ASR) weakens the communication bridges by increasing real-time delay via processing overhead. ASR also performs poorly with South African accented English. Preliminary trials have influenced backend development [5] as well as interfaces targeted for both deaf and hearing users [7].

The second community is located in a remote rural region. Tsilitwa (Eastern Cape) has a clinic without a doctor that serves roughly 10,000 people. The Centre for Scientific and Industrial Research (CSIR) has installed a wireless Ethernet network with basic voice and video over IP to allow clinic nurses to communicate with a doctor in a neighboring village. However, the system is rarely used due to frequent power outages. Visits to Tsilitwa, workshops with the CSIR and frequent communication with a local NGO called bridges.org, together have yielded software requirements for a multi-modal store-and-forward system to overcome the power problems. A prototype is under development [2].

\section{WORKPLAN}

Relationships with the communities have been developed and we have funding to carry out the fieldwork. Software prototypes have been deployed, and we are continuing to measure their effectiveness. In brief, the work remaining is to continue the cyclical interaction with the target communities to learn how to build working bridges and to perform an evaluation for each cycle.

\section{CONCLUSION}

Interaction with various stakeholders and initial field trials with two communities have yielded innovative software for communication bridges. This software appears to compensate for problems encountered when building bridges over the Digital Divide. Backed by quantitative measurement, the research shows promise for conducting HCI research specifically for the developing world, and for mainstream HCI in the process.

\section{ACKNOWLEDGMENTS}

Many thanks to the Telkom Centres of Excellence with Siemens and Cisco, the South Africa Netherlands Policy Alternatives for Development (SANPAD), bridges.org, the University of the Western Cape, Edwin Blake, Gary Marsden and Susan Dray, for their support.

\section{REFERENCES}

1. Benjamin, A.E. and Tucker W.D. Floor Control Arbitration for a Hybrid Voice/Text Web-board. Proc. South African Telecommunications Networks \& Applications Conference (SATNAC) 2003.

2. Chetty, M., Tucker, W. and Blake, E. Using Voice over IP to Bridge the Digital Divide: A Critical Action Research Approach. Proc. South African Telecommunications Networks \& Applications Conference (SATNAC) 2003.

3. Dray, S. and Siegel D. Learning from Latin America: Methodological Lessons from Emerging Markets. Proc. Contextual Invention 2003, 9-18.

4. Erickson, T. and Kellogg, W.A. Social Translucence: Using Minimalist Visualizations of Social Activity to Support Collective Interaction. Designing Information Spaces: The Social Navigation Approach, Springer Press (2003), 17-41.

5. Lewis, J., Tucker, W. and Blake, E. SoftBridge: A Multi-modal Instant Messaging Bridging System. Proc. South African Telecommunications Networks \& Applications Conference (SATNAC) 2003.

6. Nardi, B.A., Whittaker S. and Bradner, E. Interaction and Outeraction: Instant Messaging in Action. Proc. CSCW 2000, 79-88.

7. Tucker, W.D., Glaser, M. and Lewis, J. SoftBridge in Action: The First Deaf Telephony Pilot, Proc. South African Telecommunications Networks \& Applications Conference (SATNAC) 2003.

8. Whittaker, S. and Amento, B. "Seeing What You Are Hearing: Co-ordinating Responses to Trouble Reports in Network Troubleshooting", Proc. ECSCW 2003, 219238. 